Internat. J. Math. \& Math. Sci.

Vol. 22, No. 1 (1999) 13-16

S 0161-1712<99>22013-3

(c) Electronic Publishing House

\title{
BUCHWALTER-SCHMETS THEOREMS AND LINEAR TOPOLOGIES
}

\section{M. SÁNCHEZ RUIZ and J. R. FERRER}

(Received 28 January 1997)

\begin{abstract}
In this paper, we obtain Buchwalter-Schmets theorems in the realm of Lefschetz linearly topologized spaces.
\end{abstract}

Keywords and phrases. $\mu$-space, replete, barrelled, linearly topologized space.

1991 Mathematics Subject Classification. 46A15, 54D60.

1. Introduction. Throughout this paper, $X$ stands for Hausdorff completely regular topological space, $v X$ for its Hewitt real compactification, $\beta X$ for its Čech-Stone compactification, and $C(X)$ for the space of continuous functions from $X$ into the real field $\mathbb{R}$ endowed with its usual topology. Let us recall that a subset $B$ of $X$ is bounding if $f(B)$ is bounded for each $f \in C(X)$. X is said to be a $\mu$-space if each bounding subset of $X$ is relatively compact and $X$ is replete if $X=v X$. The space $C(X)$ is denoted by $C_{c}(X)$ when we consider it endowed with the compact-open topology, and by $C_{s}(X)$ when endowed with the topology of pointwise convergence.

Nachbin [5] and Shirota [13] studied the relationship between the topological properties of $X$ and $C_{c}(X)$. They showed that $X$ is a $\mu$-space if and only if $C_{c}(X)$ is barrelled and that $X$ is replete if and only if $C_{c}(X)$ is bornological. Afterwards, De WildeSchmets [2] showed the latter to be true if and only if $C_{c}(X)$ is ultrabornological. And Buchwalter-Schmets [1] studied the relationship between the topological properties of $X$ and $C_{S}(X)$ (see also [6]), obtaining:

(i) $C_{S}(X)$ is barrelled if and only if each bounding subset of $X$ is finite.

(ii) $C_{s}(X)$ is bornological if and only if $X$ is replete.

(iii) $C_{s}(X)$ is ultrabornological if and only if $X$ is replete and each compact subset of $X$ is finite.

On the other hand, Lefschetz introduced the linearly topologized spaces as those Hausdorff topological vector spaces over a discrete field with a topology which is linear (cf. [3]). In their context, we defined and studied the linearly barrelled [10], linearly bornological [8], and linearly ultrabornological [9] spaces. With them we obtained the theorems of Nachbin-Shirota and De Wilde-Schmets in the realm of linearly topologized spaces. In order to get them, we considered the linearly topologized space $C_{\lambda}(X)$, defined over the discrete field $\mathbb{R}$, generated when the vector subspaces $N_{K}=\{f \in C(X): K \cap \operatorname{supp} f=\varnothing\}$, for each compact subset $K$ of $X$, are taken to be a base of neighborhoods of the origin in $C(X)$. Then we were able to prove that $X$ is a $\mu$-space if and only if $C_{\lambda}(X)$ is linearly barrelled [10], and that $X$ is replete if and only if $C_{\lambda}(X)$ is linearly bornological [8] or linearly ultrabornological [9].

In this paper, we also extend Buchwalter-Schmets results on $C_{S}(X)$ to the setting 
of linearly topologized spaces. With this aim, we consider the linearly topologized space $C_{\sigma}(X)$, defined over the discrete field $\mathbb{R}$, obtained by endowing $C(X)$ with the topology that admits, as a base of neighborhoods of the origin, the vector subspaces $N_{K}=\{f \in C(X): K \cap \operatorname{supp} f=\varnothing\}$, for each finite subset $K$ of $X$.

Given $f \in C(X), f^{*}$ denotes the continuous extension of $f$ from $\beta X$ into the Alexandroff compactification of $\mathbb{R}$. Finally, let us recall that, for each non void absolutely convex subset $H$ of $C(X)$, there exists a minimum compact subset of $\beta X$, supp $H$, such that if $f \in C(X)$ and $\operatorname{supp} H \cap \operatorname{supp} f^{*}=\varnothing$, then $f \in H$, [12, II.1.3].

2. Buchwalter-Schmets theorems. A null sequence $\left(x_{n}\right)$ of a linearly topologized space is said to be complete [10] if, for each sequence $\left(\alpha_{n}\right) \in \omega$, the Cauchy sequence $\left(\sum_{i=1}^{n} \alpha_{i} x_{i}\right)$ is convergent. Let us recall $([10,8,9]$ respectively) that:

Definition. A linearly topologized space $L$ is said to be :

(a) linearly barrelled if each closed linear subspace $H$ of $L$ that eventually contains every complete null sequence is open.

(b) linearly bornological if each linear subspace $H$ of $L$ that eventually contains every null sequence is open.

(c) linearly ultrabornological if each linear subspace $H$ of $L$ that eventually contains every complete null sequence is open.

Clearly, every linearly ultrabornological space is linearly bornological and linearly barrelled, whilst every sequentially complete linearly bornological space is linearly ultrabornological.

Next, we characterize the open subspaces of $C_{\sigma}(X)$.

LEMMA 1. A linear subspace $L$ of $C_{\sigma}(X)$ is open if and only if $\operatorname{supp} L$ is a finite subset of $X$.

Proof. Clearly, the set $M=\left\{f \in C(X): \operatorname{supp} L \cap \operatorname{supp} f^{*}=\varnothing\right\}$ is contained in $L$. So, $M=\{f \in C(X): \operatorname{supp} L \cap \operatorname{supp} f=\varnothing\}$ is open in $C_{\sigma}(X)$ and, consequently, $L$ is open as well.

Conversely, if $L$ is open, then there exists some finite subset $K$ of $X$ such that $\{f \in$ $C(X): K \cap \operatorname{supp} f=\varnothing\}=\left\{f \in C(X): K \cap \operatorname{supp} f^{*}=\varnothing\right\} \subset L$. Hence, $\operatorname{supp} L \subset K \subset X$.

From this lemma, and the fact that if $A$ is a subset of $X$ and $L$ is the linear subspace of $C(X)$ formed by those functions vanishing on $A$, then $\operatorname{supp} L$ coincides with the closure of $A$ in $\beta X$, it follows that $L$ is an open subspace of $C_{\sigma}(X)$ if and only if $A$ is finite.

LEMMA 2. A subset $A$ of $X$ is bounding if and only if the linear subspace $L=\{f \in$ $C(X): f(A)=\{0\}\}$ is open in the strong linear topology of $C_{\sigma}(X)$.

Proof. In order to show that $L$ is open in the strong linear topology of $C_{\sigma}(X)$, it is enough to prove that each complete null sequence $\left(f_{n}\right)$ of $C_{\sigma}(X)$ is eventually contained in $L,[3, \S 10.9(1)-(3)$ and $\S 12.1(5)]$. Suppose that $\left(f_{n}\right)$ is a complete null sequence which is not contained in $L$, then there exists some $a_{1} \in A$ and $n_{1} \in \mathbb{N}$ such that $f_{n_{1}}\left(a_{1}\right) \neq 0$. So, by recurrence, for each $p \in \mathbb{N}$, there exists some $a_{p+1} \in A$ 
and $n_{p+1} \in \mathbb{N}$ such that $f_{n_{p+1}}\left(a_{i}\right)=0$, for $i<p+1$, and $f_{n_{p+1}}\left(a_{p+1}\right) \neq 0$ since $\left\{a_{1}, a_{2}, \ldots, a_{p}\right\}$ is finite. On the other hand, $\left\{f_{n_{p}}: p \in \mathbb{N}\right\}$ being a complete null sequence, it implies that the function $g:=\sum_{p=1}^{\infty} \alpha_{p} f_{n_{p}} \in C(X)$ for each $\left(\alpha_{n}\right) \in \omega$. Thus, if we select the $\alpha_{p}$ in such a way that $g\left(a_{p}\right) \geq p$, we conclude that $A$ is not bounding. Contradiction.

Conversely, if $A$ is not bounding, then there is some $g \in C(X)$ and a sequence $\left(a_{n}\right)$ in $A$ such that $\left|g\left(a_{n}\right)\right|>1+\left|g\left(a_{n-1}\right)\right|$. Let us consider a sequence $\left(f_{n}\right)$ in $C(X)$ such that $f_{n}\left(a_{n}\right)=1$ and $\operatorname{supp} f_{n} \subseteq\left\{x \in X:\left|g\left(a_{n}\right)-g(x)\right|<1 / 2\right\}$. Then $f_{n} \in C(X) \backslash L$ for every $n \in \mathbb{N}$ and $L$ is not open in the strong linear topology of $C_{\sigma}(X)$ since $\left\{\operatorname{supp} f_{n}: n \in \mathbb{N}\right\}$ is locally finite and, consequently, $\left(f_{n}\right)$ is a complete null sequence.

The following results include Buchwalter-Schmets theorems for linearly topologized spaces (see [7] for additional information on linear inductive limits).

THEOREM 1. Let $X$ be a Hausdorff completely regular topological space. Then $C_{\sigma}(X)$ is linearly barrelled if and only if each bounding subset of $X$ is finite.

Proof. Assume that there exists a bounding subset $A$ of $X$ which is not finite. Since $C_{\sigma}(X)$ is endowed with the strong linear topology [10], by Lemma 2, the linear subspace $L=\{f \in C(X): f(A)=\{0\}\}$ is open in $C_{\sigma}(X)$ and Lemma 1 implies that $\operatorname{supp} L$, which coincides with the closure of $A$ in $\beta X$, is a finite subset of $X$. This contradicts the fact that $A$ is not finite. Conversely, if each bounding subset of $X$ is finite, $C_{\sigma}(X)$ coincides with $C_{\lambda}(X)$ and $X$ is a $\mu$-space. Hence, $C_{\sigma}(X)$ is linearly barrelled [10].

THEOREM 2. Let X be a Hausdorff completely regular topological space. The following assertions are equivalent:

(i) $X$ is replete.

(ii) $C_{\sigma}(X)$ is a linearly bornological space.

(iii) $C_{\sigma}(X)$ is the linear inductive limit of the family formed by its countable-dimensional subspaces.

(iv) Each linear form defined on $C_{\sigma}(X)$, that has a continuous restriction to each countable-dimensional subspace, is continuous.

Proof. [(i) $\Rightarrow$ (ii)] Let $L$ be a linear subspace of $C_{\sigma}(X)$ which contains eventually each null sequence. By [10, Lem. 4] $\operatorname{supp} L \subset v X=X$. If $\operatorname{supp} L$ were not finite, then there would exist a sequence $\left(a_{n}\right)$ in $\operatorname{supp} L$ and a sequence $\left(U_{n}\right)$ of pairwise disjoint open neighborhoods in $\beta X$ such that $a_{n} \in U_{n}$ [11, II.11.6]. Then, for each $n \in \mathbb{N}$, there exists some $f_{n} \in C(X) \backslash L$ whose $\operatorname{supp} f_{n}^{*} \subset U_{n}$. And from the fact that $\left\{\operatorname{supp} f_{n}: n \in \mathbb{N}\right\}$ are pairwise disjoint, it follows that $\left(f_{n}\right)$ is a null sequence. This is not possible since $f_{n} \notin L$ for each $n \in \mathbb{N}$.

Finally, [(ii) $\Rightarrow($ iii)] follows from [8, Prop. 1], [(iii)] $\Rightarrow$ (iv)] is pretty obvious and the argument used in [8, Thm. 1] shows [(iv) $\Rightarrow$ (i)].

THEOREM 3. Let $X$ be a Hausdorff completely regular topological space. The following assertions are equivalent:

(i) $X$ is replete and each compact subset of $X$ is finite.

(ii) $C_{\sigma}(X)$ is a linearly ultrabornological space. 
Proof. [(i) $\Rightarrow$ (ii)] If each compact subset of $X$ is finite, then $C_{\sigma}(X)$ coincides with $C_{\lambda}(X)$ and since $X$ is replete, [9] gives (ii).

$[(\mathrm{ii}) \Rightarrow(\mathrm{i})]$ is clear.

ACKNOWLEDGEMENT. Supported by DGES PB97-0342 and IVEI 03-033.

\section{REFERENCES}

[1] H. Buchwalter and J. Schmets, Sur quelques propriétés de l'espace $C_{S}(T)$, J. Math. Pures Appl. 52 (1973), no. 9, 337-352. MR 48 12012. Zbl 268.46025.

[2] M. De Wilde and J. Schmets, Caractérisation des espaces $C(X)$ ultrabornologiques, Bull. Soc. Roy. Sci. Liège 40 (1971), 119-121. MR 45 872. Zbl 216.41001.

[3] G. Kothe, Topological vector spaces. I, Translated from the German by D. J. H. Garling. Die Grundlehren der mathematischen Wissenschaften, Springer-Verlag New York Inc., New York, 1969. MR 40\#1750. Zbl 179.17001.

[4] S. Lefschetz, Algebraic Topology, American Mathematical Society Colloquium Publications, vol. 27, American Mathematical Society, New York, 1942. MR 4,84f. Zbl 061.39302.

[5] L. Nachbin, Topological vector spaces of continuous functions, Proc. Nat. Acad. Sci. U.S.A 40 (1954), 471-474. MR 16,156h. Zbl 055.09803.

[6] L. M. S. Ruiz, On Buchwalter and Schmets' theorems, Zb. Rad. Prirod. Mat. Fak. 23 (1993), no. 1, 51-55. MR 96a:46045. Zbl 824.46002.

[7] L. M. S. Ruiz and J. C. Ferrando, Límites inductivos de espacios linealmente topologizados, Proceedings of the XIVth Spanish-Portuguese Conference on Mathematics, vol. I-III, Univ. La Laguna, 1989, pp. 547-551. CMP 9114.

[8] L. M. S. Ruiz and M. López Pellicer, On linearly topologized spaces and real-compact spaces. I, Portugal. Math. 48 (1991), no. 4, 397-404. MR 93h:46003. Zbl 776.46004.

[9] _ On linearly topologized spaces and real-compact spaces. II, Portugal. Math. 48 (1991), no. 4, 475-482. MR 93h:46003. Zbl 776.46005.

[10] L. M. S. Ruiz and M. L. Pellicer, On linearly topologized spaces and $\mu$-spaces, Portugal. Math. 48 (1991), no. 3, 309-318. MR 92k:46011. Zbl 795.46005.

[11] J. Schmets, Espaces de fonctions continues, vol. 519, Springer-Verlag, Berlin, New York, 1976, Lecture Notes in Mathematics. MR 54 11041. Zbl 334.46022.

[12] _ Spaces of vector-valued continuous functions, Lecture Notes in Mathematics, vol. 1003, Springer-Verlag, Berlin, New York, 1983. MR 85g:46046. Zbl 511.46033.

[13] T. Shirota, On locally convex vector spaces of continuous functions, Proc. Japan Acad. 30 (1954), 294-298. MR 16,275d. Zbl 057.33801.

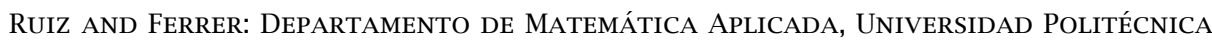
De VALENCIA, E-46022, VALENCIA, SPAin 


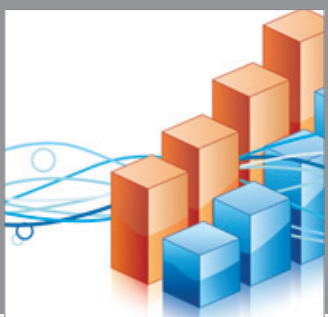

Advances in

Operations Research

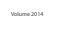

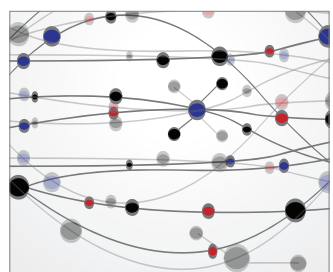

\section{The Scientific} World Journal
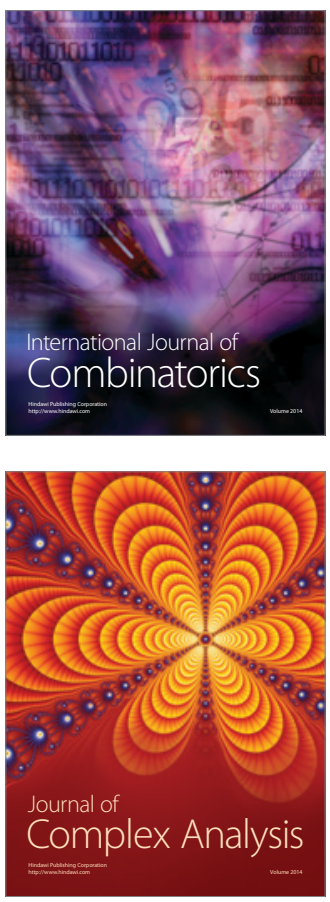

International Journal of

Mathematics and

Mathematical

Sciences
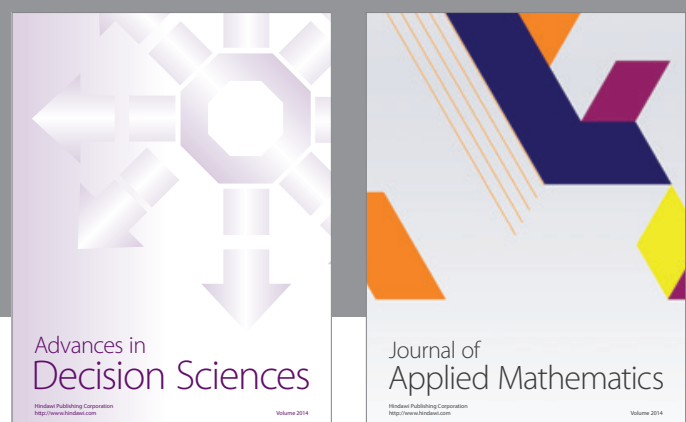

Journal of

Applied Mathematics
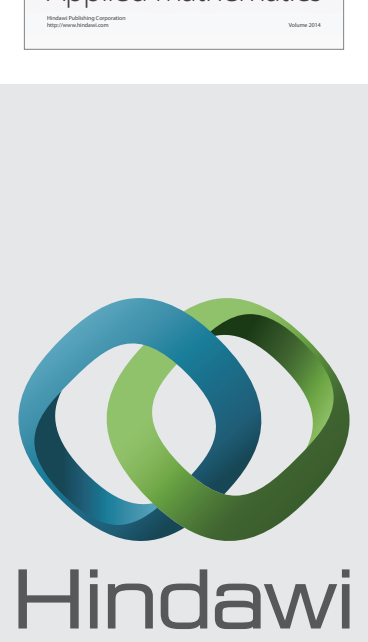

Submit your manuscripts at http://www.hindawi.com
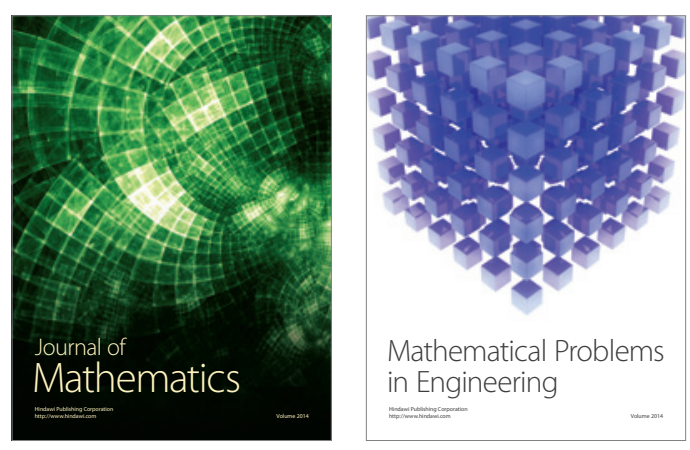

Mathematical Problems in Engineering
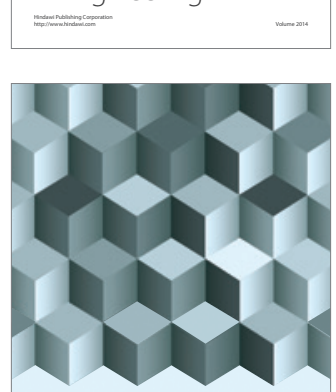

Journal of

Function Spaces
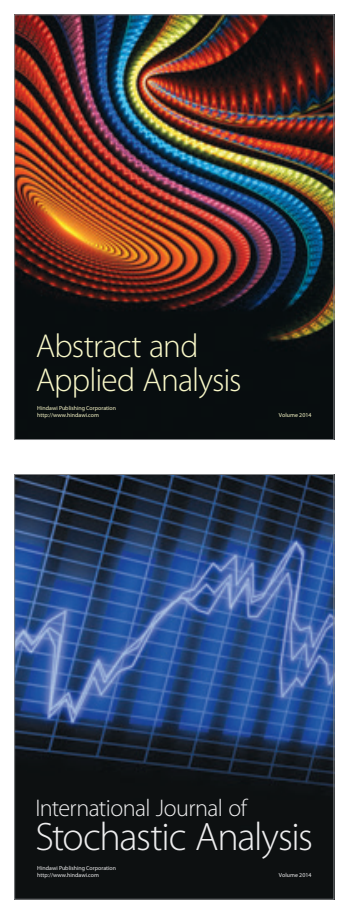

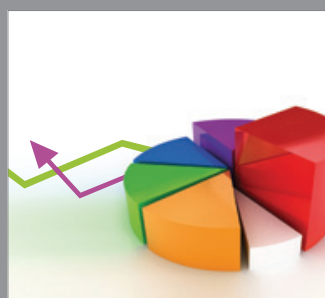

ournal of

Probability and Statistics

Promensencen
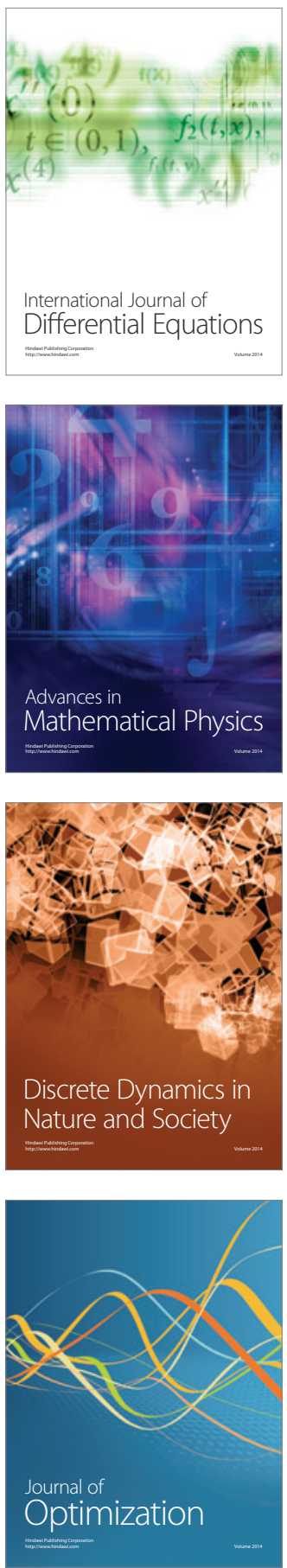